\title{
Emerging treatments and combinations in the management of NSCLC: clinical potential of nintedanib
}

\author{
Martin Reck' \\ Anders Mellemgaard ${ }^{2}$ \\ 'Department of Thoracic Oncology, \\ Lung Clinic Grosshansdorf, and \\ member of the Airway Research \\ Center North, German Center \\ for Lung Research, Grosshansdorf, \\ Germany; '2Department of Oncology, \\ Herlev University Hospital, \\ Copenhagen, Denmark
}

This article was published in the following Dove Press journal:

Biologics: Targets and Therapy

I July 2015

Number of times this article has been viewed

REVIEW

\begin{abstract}
There remains an unmet need for effective, well-tolerated treatment options in advanced non-small cell lung cancer (NSCLC) to alleviate the disease burden for a broad selection of patients. Nintedanib is a potent, oral, triple angiokinase inhibitor of vascular endothelial growth factor, fibroblast growth factor, and platelet-derived growth factor, and was recently approved in Europe for use in combination with docetaxel for the treatment of adults with locally advanced, metastatic, or locally recurrent NSCLC of adenocarcinoma tumor histology, following first-line chemotherapy. Nintedanib has been investigated extensively in preclinical research and in a number of clinical studies, the most important of which was the Phase III LUME-Lung 1 study, which investigated nintedanib in combination with docetaxel in patients with advanced NSCLC after failure of first-line chemotherapy. In this study, which led to the approval of nintedanib, addition of nintedanib to docetaxel significantly improved overall survival in patients with adenocarcinoma histology. Nintedanib demonstrated a manageable safety profile in combination with docetaxel. This review focuses on the clinical experience with nintedanib in NSCLC and discusses the clinical potential of this agent for use in combination with chemotherapy.
\end{abstract}

Keywords: nintedanib, non-small cell lung cancer, second-line treatment, docetaxel, adenocarcinoma, antiangiogenesis

\section{Introduction}

Lung cancer is the most commonly diagnosed cancer, with an estimated incidence of 1.8 million new cases in 2012, representing $12.9 \%$ of all new diagnoses. ${ }^{1}$ Lung cancer is also the leading cause of cancer mortality in the world, with a mortality rate of nearly one in five (1.59 million deaths), which equates to $19.4 \%$ of all cancer deaths. ${ }^{1}$ Non-small cell lung cancer (NSCLC) is the most frequent type of lung cancer, accounting for more than $85 \%$ of all cases. NSCLC is composed of different histological types, which are divided into two main groups that guide treatment strategy: non-squamous carcinoma (comprising adenocarcinoma, large-cell carcinoma, and other cell types) and squamous cell carcinoma. ${ }^{2}$

Proangiogenic pathways have been established as important and effective therapeutic targets in many cancers, including NSCLC, because they are essential for tumor growth, progression, and metastasis. ${ }^{3}$ Bevacizumab, a monoclonal antibody that targets vascular endothelial growth factor (VEGF), was the first antiangiogenic agent approved for the treatment of non-squamous NSCLC as first-line therapy in combination with platinum-based chemotherapy. ${ }^{2,4,5}$

Platinum doublet-based chemotherapy, with or without bevacizumab, is currently the standard first-line treatment option for patients with advanced NSCLC and no
Correspondence: Martin Reck Department of Thoracic Oncology, Lung Clinic Grosshansdorf,

Wohrendamm 80, 22927 Grosshansdorf, Germany

Tel +4941026012101

Fax +494102601247

Email dr.martin.reck@web.de (c) (i) (-) 2015 Reck and Mellemgaard. This work is published by Dove Medical Press Limited, and licensed under Creative Commons Attribution - Non Commercial (unported, v3.0) License. The full terms of the License are available at http://creativecommons.org/licenses/by-nc/3.0/. Non-commercial uses of the work are permitted limite are Limited. Information on how to request permission may be found at: http://www.dovepress.com/permissions.php 
actionable biomarker. ${ }^{2,4,5}$ However, approximately $25 \%$ of patients with advanced NSCLC show disease progression after the initial cycle of first-line chemotherapy, and most patients will eventually experience relapse and disease progression, requiring second-line treatment. ${ }^{2,6,7}$ Furthermore, a substantial number of patients are either intrinsically refractory or develop resistance to bevacizumab-based regimens. ${ }^{8}$

Agents that are currently recommended for second-line treatment of NSCLC include the cytotoxic agents docetaxel and pemetrexed (the latter for patients with non-squamous histology), and the tyrosine kinase inhibitor erlotinib for patients who are refractory to chemotherapy. ${ }^{4,5}$ Despite a significant amount of research over the last 10 years, no improvements in overall survival compared with standard treatment had been reported and, until the LUME-Lung 1 study, no combination regimens investigated showed improvements in overall survival in the second-line setting. ${ }^{9-16}$

One strategy for improving efficacy, as well as overcoming therapeutic resistance to bevacizumab, is to target multiple angiogenic pathways. Nintedanib (Vargatef ${ }^{\circledR}$; Boehringer Ingelheim Ltd, Ingelheim, Germany) is a triple angiokinase inhibitor (https://youtu.be/0gDnGFumAXE) that simultaneously inhibits signaling pathways activated by
VEGF, fibroblast growth factor (FGF), and platelet-derived growth factor (PDGF). In November 2014, nintedanib was approved in Europe for use in combination with docetaxel for the treatment of adults with locally advanced, metastatic or locally recurrent NSCLC of adenocarcinoma histology after first-line chemotherapy. ${ }^{17}$

The aim of the present article is to review the clinical data to date for nintedanib in the second-line treatment of NSCLC in terms of efficacy, safety, and quality of life, and to explore the results of preliminary translational studies that may provide a rationale for future investigations.

\section{Pharmacology of nintedanib Mechanism of action and pharmacodynamics}

Nintedanib is a 6-methoxycarbonyl-substituted indolinone derivative that was identified in a chemical lead optimization program for ATP-competitive inhibitors of VEGF receptor 2 and other proangiogenic receptor tyrosine kinases. ${ }^{18-20}$ The binding of nintedanib to VEGF receptor 2 was confirmed by X-ray crystallography and was shown to occur in the intracellular ATP-binding site in the cleft between the $\mathrm{NH}_{2}$ and $\mathrm{COOH}$ terminal lobes of the kinase domain (Figure 1). ${ }^{18}$

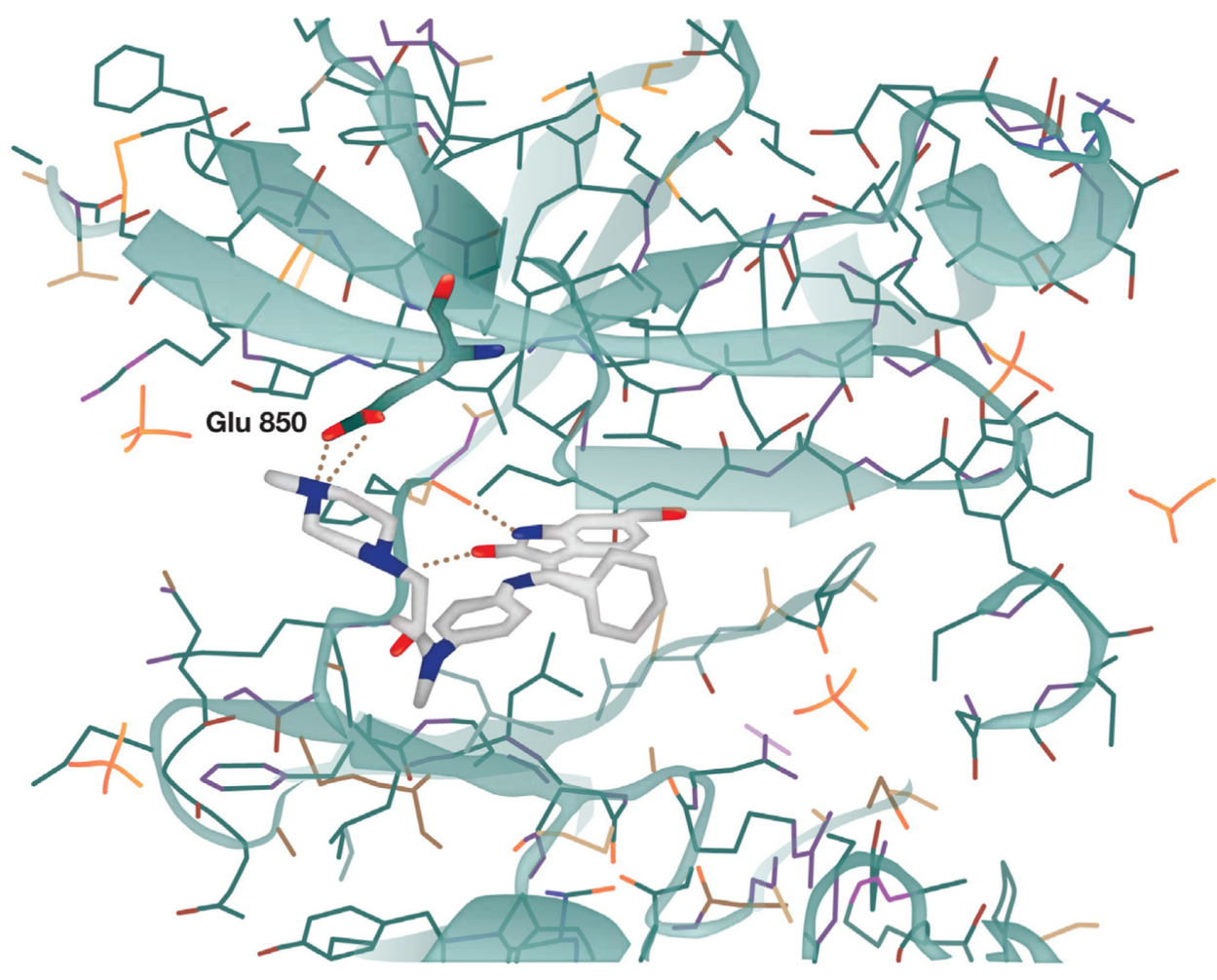

Figure I X-ray structure of nintedanib bound in the active site of the vascular endothelial growth factor receptor 2 crystal.

Note: Reprinted from Cancer Research, 2008;68(I2):4774-4782, Hilberg F, Roth GJ, Krssak M, et al, BIBF I I20: triple angiokinase inhibitor with sustained receptor blockade and good antitumor efficacy, with permission from AACR. ${ }^{18}$ 


\section{Selective kinase inhibition profile}

Extensive biochemical characterization of nintedanib showed a potent and specific inhibition of the VEGF, FGF, and PDGF receptors, and low cross-reactivity with other human kinases. ${ }^{19}$ Preclinical evidence indicated that inhibition of these kinases occurs through competitive inhibition, with nintedanib occupying the ATP-binding sites of the target kinases, thereby preventing their activation. ${ }^{18}$ Further preclinical characterization of nintedanib through in vitro inhibition assays revealed that nintedanib inhibits a distinctive, narrow range of kinases at pharmacologically relevant concentrations (Table 1). ${ }^{18}$ The targeted kinases include all three VEGF receptor subtypes (half-maximal inhibitory concentration $\left.\left[\mathrm{IC}_{50}\right] 13-34 \mathrm{nmol} / \mathrm{L}\right), \mathrm{FGF}$ receptor types 1 , 2 , and $3\left(\mathrm{IC}_{50} 69,37\right.$, and $108 \mathrm{nmol} / \mathrm{L}$, respectively), and PDGF receptor- $\alpha$ and receptor- $\beta\left(\mathrm{IC}_{50} 59\right.$ and $65 \mathrm{nmol} / \mathrm{L}$,

Table I Summary of in vitro kinase inhibition profile of nintedanib

\begin{tabular}{|c|c|}
\hline Kinase & $I C_{50}(\mathrm{nmol} / \mathrm{L})^{\mathrm{a}}$ \\
\hline VEGFR-I & $34 \pm 15$ \\
\hline VEGFR-2 & $21 \pm 13$ \\
\hline VEGFR-2 (mouse) & $13 \pm 4$ \\
\hline VEGFR-3 & $13 \pm 10$ \\
\hline FGFR-I & $69 \pm 70$ \\
\hline FGFR-2 & $37 \pm 2$ \\
\hline FGFR-3 & $|08 \pm 4|$ \\
\hline FGFR-4 & $610 \pm 117$ \\
\hline PDGFR- $\alpha$ & $59 \pm 71$ \\
\hline PDGFR- $\beta$ & $65 \pm 7$ \\
\hline InsR & $>4,000$ \\
\hline IGF-IR & $>1,000$ \\
\hline EGFR & $>50,000$ \\
\hline HER2 & $>50,000$ \\
\hline CDKI & $>10,000$ \\
\hline CDK2 & $>10,000$ \\
\hline CDK4 & $>10,000$ \\
\hline Flt-3 & 26 \\
\hline Lck & $16 \pm 16$ \\
\hline Lyn & $195 \pm 12$ \\
\hline Src & $156 \pm 40$ \\
\hline Other kinases $^{\mathrm{b}}(\mathrm{n}=26)$ & $>10,000$ \\
\hline
\end{tabular}

Notes: assays performed with ATP concentrations at the respective $K_{m}$. Human kinases were tested except when stated otherwise. Data represent mean \pm SE of at least three determinations; $I C_{50}$ values "greater than" indicate that $I C_{50}$ was not achieved at the highest concentration tested; banother 26 kinases were analyzed at $10 \mu \mathrm{mol} / \mathrm{L}$ with $100 \mu \mathrm{mol} / L$ ATP: GSK3B, ROCKII, DYRKIA, PKCA, MAPK2ERK2, HGFR, MSKI, PDKI, CHKI, MAPKAPK2, SAPK2AP38, S6KI, SGK, CKI, CK2, PKA, SAPK2BP38B2, SAPK3P38G, JNKIAI, SAPK4P38D, PHK, PKBA, CSK, CDK2/CYCLINA, PRAK, and PP2A. Adapted from Cancer Research, 2008;68(I2):4774-4782, Hilberg F, Roth G], Krssak M, et al, BIBF I I20: triple angiokinase inhibitor with sustained receptor blockade and good antitumor efficacy, with permission from AACR. ${ }^{18}$

Abbreviations: $I_{50}$, half-maximal inhibitory concentration; SE, standard error; PDGFR, platelet-derived growth factor receptor; VEGFR, vascular endothelial growth factor receptor; FGFR, fibroblast growth factor receptor; EGFR, epidermal growth factor receptor; IGF-IR, insulin-like growth factor-I receptor; InsR, insulin receptor; HER2, human epidermal growth factor receptor 2; CDK, cyclin-dependent kinase. respectively). ${ }^{18}$ The ability of nintedanib to simultaneously target these three receptor classes, thereby inhibiting alternative proangiogenic pathways, may enhance its antitumor effects and overcome pathways of resistance to VEGF/VEGF receptor 2-targeted agents. ${ }^{18}$ In addition to its triple angiokinase activity, nintedanib inhibits FLT3 and members of the Src-family (Src, Lyn, and Lck), ${ }^{18}$ but overall the inhibitor spectrum is fairly restricted, and other kinases such as insulin-like growth factor 1 receptor and epidermal growth factor receptor are not inhibited. ${ }^{18}$

The cellular effects of nintedanib on growth-dependent proliferation were observed in three cell types contributing to angiogenesis, ie, endothelial cells, smooth muscle cells, and pericytes, which are driven by VEGF, PDGF, or FGF, but not in malignant epithelial cancer cells. ${ }^{18}$ Nintedanib has been shown to inhibit proliferation and apoptosis of VEGF-stimulated endothelial cells derived from the umbilical vein and skin microvessels (half-maximal effective concentration $<10 \mathrm{nmol} / \mathrm{L}) .{ }^{18}$ Nintedanib has also been shown to inhibit the proliferation of PDGF-stimulated pericytes, which are important for vessel maturation and stabilization, and human vascular smooth muscle cells. ${ }^{18}$ However, nintedanib appears to have no effect on the proliferation of human epithelial cancer cell lines that do not express detectable VEGF receptor, FGF receptor, or PDGF receptor levels. ${ }^{18}$ In the three cell types contributing to angiogenesis, the inhibition of cell proliferation and apoptosis was preceded by inhibition of mitogen-activated protein kinase and Akt signaling pathways. ${ }^{18}$

\section{In vivo characterization}

In vivo studies in human tumor xenografts grown in nude mice and a syngeneic rate tumor model showed that nintedanib was highly active at well-tolerated doses, with both rapid changes in tumor vessel architecture, such as reduction of vessel density and integrity, and a profound induction of growth inhibition. Intracellularly, the inhibitory effect of nintedanib on VEGF receptor 2 was found to be markedly sustained, with inhibition of receptor activation for at least 32 hours after being treated for 1 hour with nintedanib, suggesting slow receptor dissociation kinetics and sustained inhibition. ${ }^{18}$

\section{In vivo effects of nintedanib on tumor vessel architecture}

Nintedanib significantly reduced tumor perfusion and vascular permeability in vivo in $\mathrm{FaDu}$ (head and neck squamous cell carcinoma) xenografts, as detected by dynamic contrast-enhanced magnetic resonance imaging 3 days after 
treatment. ${ }^{18}$ Five-day treatment of $\mathrm{FaDu}$ xenografts with nintedanib led to a significant reduction in tumor microvessel density and in PDGF receptor- $\beta$-expressing cells in the perivascular compartment compared with control tumors. Moreover, changes in tumor vessel architecture were evident from the intratumoral dissociation of endothelial cells and pericytes in xenograft tumors treated with nintedanib. ${ }^{18}$

\section{In vivo antitumor activity of nintedanib}

When tested in several cancer xenografts, nintedanib showed significant antitumor activity and good tolerability in a range of tumor types, including NSCLC (Calu-6 cells), and human renal cell (Caki-1), colorectal (HT-29), ovarian (SKOV-3), and prostate (PAC-120) carcinomas. ${ }^{18}$ Nintedanib in NSCLC model xenografts also demonstrated additive effects in combination with the cytotoxic drugs docetaxel or pemetrexed. ${ }^{21}$ Nintedanib alone and in combination with standard chemotherapy inhibited proliferation and increased apoptosis in in vivo experiments in A549 NSCLC xenografts that were poor responders to bevacizumab and resistant to platinum doublet chemotherapy. ${ }^{22}$ Further characterization of nintedanibtreated A549 NSCLC xenografts showed that nintedanib does not elevate epithelial-to-mesenchymal transition markers, suggesting that it does not drive an invasive phenotype, as has been proposed for agents that target angiogenesis. ${ }^{22}$

\section{Pharmacokinetics}

Pharmacokinetic data from healthy individuals indicate that nintedanib is rapidly absorbed following oral administration, with a median time to maximum plasma concentration of 1.3 hours and a terminal half-life of 13.7 hours. ${ }^{23}$

The major route of elimination of nintedanib is through metabolism, with its metabolites excreted via the biliary system into the feces; urinary excretion is minor $(<1 \%) .{ }^{23}$ Nintedanib metabolism in healthy humans occurs predominantly by cleavage of the methyl ester moiety, yielding the carboxylate BIBF 1202 (metabolite 1). ${ }^{23}$ BIBF 1202 is then conjugated to glucuronic acid, yielding 1-O-acylglucuronide (metabolite 2). ${ }^{23}$ Thus, metabolism of nintedanib is predominantly cytochrome P450 enzyme-independent, ${ }^{23}$ which may facilitate the combination of nintedanib with cytotoxic chemotherapies, such as docetaxel, that are metabolized via cytochrome P450 enzymes. ${ }^{23}$

\section{Dose-finding and drug-drug interaction studies}

Based on several Phase I nintedanib monotherapy doseescalation studies in patients with a variety of advanced solid tumors, the maximum tolerated dose of nintedanib was defined as $250 \mathrm{mg}$ twice daily in Caucasian patients and $200 \mathrm{mg}$ twice daily in Japanese patients. ${ }^{24-26}$

Two Phase I studies evaluated the maximum tolerated dose of nintedanib in combination with cytotoxic therapy, as well as potential drug-drug interactions. One Phase I study in 26 patients with advanced NSCLC evaluated the effects of first-line combination therapy with nintedanib (50-250 mg twice daily) on days 2-21, plus paclitaxel (200 mg/m² body surface area) and carboplatin (area under the curve $6 \mathrm{mg} / \mathrm{mL} / \mathrm{min}$ ) on day 1 of each 21-day cycle, and found that nintedanib had no clinically relevant influence on the pharmacokinetics of paclitaxel or carboplatin and vice versa. ${ }^{27}$ A second Phase I study of 26 patients, which examined the tolerability of nintedanib (100-250 mg twice daily) on days $2-21$ in combination with pemetrexed $\left(500 \mathrm{mg} / \mathrm{m}^{2}\right)$ on day 1 as second-line treatment following platinum-based chemotherapy in patients with advanced NSCLC, found no clinically relevant pharmacokinetic interactions between nintedanib and pemetrexed. ${ }^{28}$ In both combination regimens, the maximum tolerated dose was established as nintedanib $200 \mathrm{mg}$ twice daily. ${ }^{27,28}$

In a third Phase I study, which assessed the tolerability of nintedanib (100-200 mg twice daily on days 2-21) in combination with docetaxel (60 or $75 \mathrm{mg} / \mathrm{m}^{2}$ on day 1) as second-line therapy for advanced NSCLC in Japanese patients $(n=42)$, pharmacokinetic analysis revealed no apparent interactions between nintedanib and docetaxel. ${ }^{29}$ The maximum tolerated dose of nintedanib in combination with docetaxel in Japanese patients was dependent on body surface area: $150 \mathrm{mg}$ twice daily for a body surface area $<1.5 \mathrm{~m}^{2}$ and $200 \mathrm{mg}$ twice daily for a body surface area $\geq 1.5 \mathrm{~m}^{2} .^{29}$

\section{Clinical studies \\ Phase II study}

The safety and efficacy of nintedanib monotherapy (150 or $250 \mathrm{mg}$ twice daily) in patients with locally advanced or metastatic relapsed NSCLC requiring second-line or thirdline therapy were evaluated in a double-blind Phase II study. Primary endpoints assessed were progression-free survival and objective tumor response (Response Evaluation Criteria In Solid Tumors). ${ }^{30}$ In total, 73 patients were enrolled and randomized: 37 to nintedanib $150 \mathrm{mg}$ twice daily and 36 to nintedanib $250 \mathrm{mg}$ twice daily. ${ }^{30}$

The median progression-free survival for all patients was 6.9 weeks, with no significant difference between the two nintedanib dose groups. ${ }^{30}$ The median progression-free survival was significantly longer in patients with baseline 
Eastern Cooperative Oncology Group (ECOG) performance status $0-1$ versus patients with ECOG performance status of 2 (11.6 versus 6 weeks; hazard ratio [HR] 3.19; $P=0.0002)$; subgroup analyses showed no difference in progression-free survival between patients with squamous versus non-squamous NSCLC. ${ }^{30}$ The median overall survival for all patients was 21.9 weeks; the median overall survival for patients with ECOG 0-1 was 37.7 weeks. ${ }^{30}$ One patient in the higher-dose nintedanib group achieved a confirmed partial response and a total of 35 patients (48\%) achieved stable disease. ${ }^{30}$

Continuous daily treatment with nintedanib was generally well tolerated. ${ }^{30}$ Most adverse events reported in this study were mild to moderate in nature and predominantly gastrointestinal (ie, nausea, diarrhea, and vomiting). ${ }^{30}$ Grade 3 or worse adverse events were observed at a higher frequency in the higher nintedanib dose versus the lower nintedanib dose group $(41.7 \%$ versus $8.1 \%) .{ }^{30}$ The incidence of fatigue and adverse events associated with other VEGF receptor-targeted agents, such as hypertension, bleeding, and thromboembolic events, was low, and no patients developed hand-foot syndrome. ${ }^{30}$ The overall safety pattern was similar for patients with squamous and non-squamous histology. ${ }^{30}$

\section{Phase III studies}

The Phase III clinical development program for nintedanib in NSCLC consisted of two studies that evaluated nintedanib in combination with cytotoxic therapy, ie, LUME-Lung $1^{31}$ and LUME-Lung $2 .^{32}$

LUME-Lung 1 was the pivotal trial that led to approval of nintedanib in combination with docetaxel. This multinational, randomized, placebo-controlled trial (study 1199.13, NCT00805194) assessed the efficacy and safety of nintedanib (200 mg twice daily on days 2-21) plus docetaxel $\left(75 \mathrm{mg} / \mathrm{m}^{2}\right.$ on day 1) as second-line therapy for advanced stage IIIB/IV, recurrent NSCLC of all histologies progressing after first-line chemotherapy. ${ }^{31}$ The primary endpoint was progressionfree survival by independent central review, which was analyzed after 714 events in all patients. ${ }^{31}$ Overall survival was predefined as a key secondary outcome and analyzed on an intention-to-treat basis in a prespecified, stepwise, fixed-sequence order: first, in a predefined group of patients with adenocarcinoma and poor prognosis (defined as time elapsed since start of first-line therapy of less than 9 months until randomization into the trial); second, in patients with adenocarcinoma; and finally, in all patients regardless of histology. ${ }^{31}$ A total of 1,314 patients from 211 centers in 27 countries were randomized to receive study treatment:
655 patients were assigned to nintedanib plus docetaxel and 659 to placebo plus docetaxel. ${ }^{31}$

The primary study endpoint of LUME-Lung 1 was met; progression-free survival was significantly improved in the nintedanib plus docetaxel group compared with the placebo plus docetaxel group (3.4 months [95\% confidence interval (CI) 2.9-3.9] versus 2.7 months [95\% CI 2.6-2.8]; HR 0.79 [95\% CI 0.68-0.92]; $P=0.0019) .{ }^{31}$ Similar results were noted in patients with adenocarcinoma and patients with squamous cell carcinoma. ${ }^{31}$ In the adenocarcinoma population, the median overall survival was greater than 1 year in the nintedanib plus docetaxel group and significantly greater than in the placebo plus docetaxel group (12.6 months [95\% CI 10.6-15.1] versus 10.3 months [95\% CI 8.6-12.2]; HR 0.83 [95\% CI 0.70-0.99]; $P=0.0359$, Figure 2). ${ }^{31}$ Improvement was also observed in patients with adenocarcinoma histology who progressed rapidly, within 9 months of the start of first-line therapy; the median overall survival was 10.9 months with nintedanib plus docetaxel versus 7.9 months with placebo plus docetaxel (HR 0.75, 95\% CI 0.60-0.92; $P=0.0073$, Figure 2). ${ }^{31,33}$ For both the overall adenocarcinoma population and the adenocarcinoma patients who progressed rapidly, the probability of survival at 12 and 24 months was greater for patients treated with nintedanib plus docetaxel versus patients treated with placebo plus docetaxel. In the overall adenocarcinoma population, 12 month overall survival was $52.7 \%$ with nintedanib plus docetaxel compared with $44.7 \%$ with placebo and docetaxel, and 24 month overall survival was $25.7 \%$ compared with $19.1 \%$. In the adenocarcinoma population of patients who progressed rapidly, 12 month overall survival was $46.8 \%$ with nintedanib plus docetaxel versus $34.3 \%$ with placebo plus docetaxel; 24 month overall survival was $20.7 \%$ versus $10.4 \%$. An exploratory analysis was also conducted in patients with adenocarcinoma refractory to first-line treatment chemotherapy. These patients had achieved only progressive disease as the best response to first-line therapy prior to entry into the LUME-Lung 1 trial. ${ }^{31,33}$ The median overall survival was 9.8 months with nintedanib plus docetaxel versus 6.3 months with placebo plus docetaxel (HR 0.62, 95\% CI 0.41-0.94; $P=0.0246$, Figure 3 ) in this subpopulation; the probability of survival was higher in patients treated with nintedanib plus docetaxel versus placebo plus docetaxel at 12 months $(43.0 \%$ versus $24.6 \%)$ and 24 months $(21.5 \%$ versus $5.3 \%){ }^{31}$

Despite a numerical improvement, overall survival with nintedanib plus docetaxel in the total study population was not statistically significant (median 10.1 months [95\% CI 
8.8-11.2] versus 9.1 months [95\% CI 8.4-10.4]; HR 0.94, $95 \%$ CI 0.83-1.05; $P=0.2720$, Figure 2). ${ }^{31}$

A second study, the multinational, randomized, doubleblind, placebo-controlled LUME-Lung 2 trial (study 1199.14; NCT00806819) evaluated the efficacy and safety of nintedanib (200 mg twice daily on days 2-21) plus standard pemetrexed $\left(500 \mathrm{mg} / \mathrm{m}^{2}\right.$ on day 1$)$ as second-line therapy for
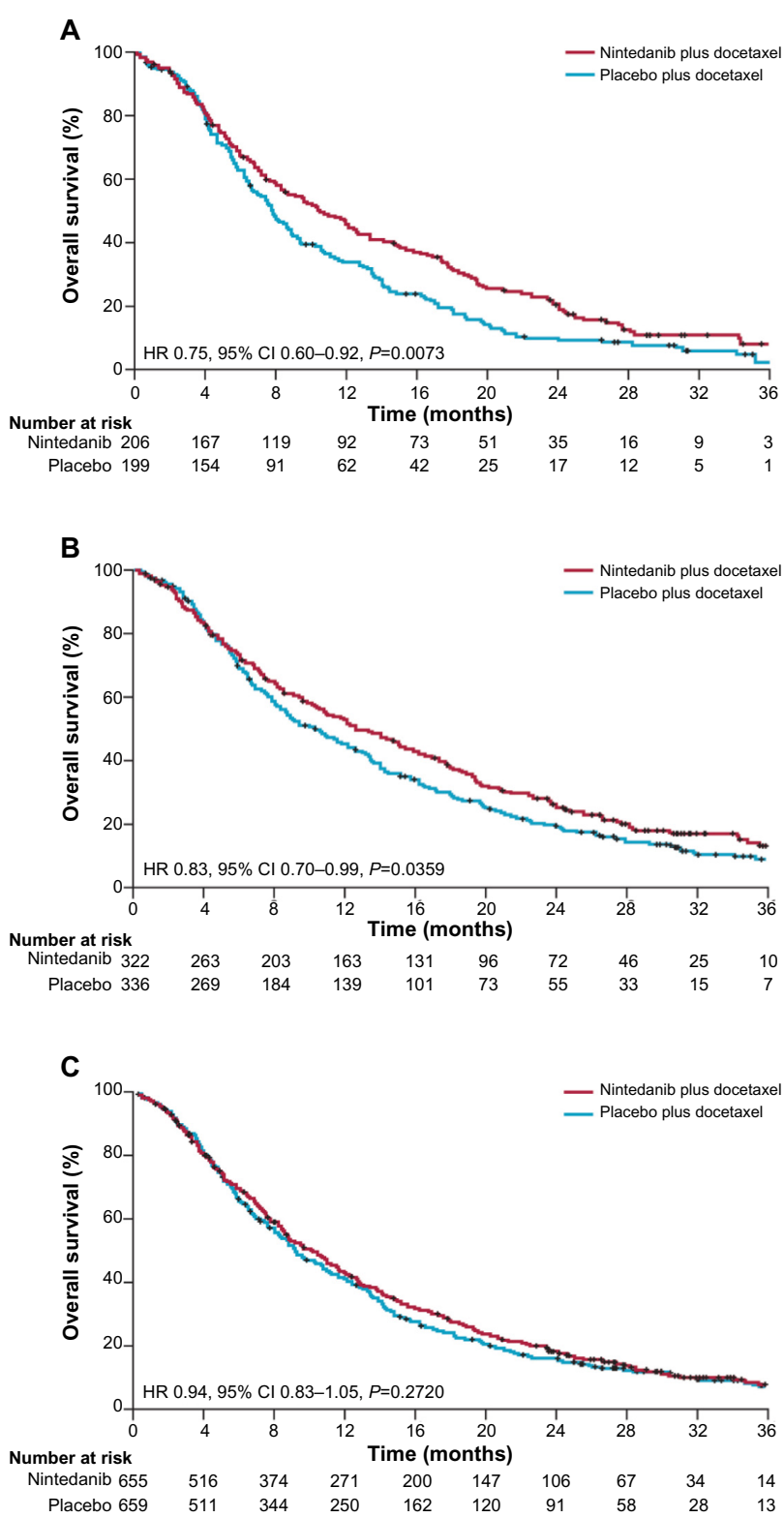

Figure 2 Kaplan-Meier curves for patients treated with nintedanib plus docetaxel or placebo plus docetaxel.

Notes: Overall survival at the time of final analysis in (A) patients with adenocarcinoma histology and time since start of first-line therapy of less than 9 months; (B) all patients with adenocarcinoma histology; (C) total population. Patients without documented death were censored at the date of last contact when the patient was known to be alive. Reprinted from Lancet Oncol, Vol I5, Reck M, et al, Docetaxel plus nintedanib versus docetaxel plus placebo in patients with previously treated non-small cell lung cancer (LUME-Lung I): a phase 3, double-blind, randomised trial, 143-I55, Copyright (2014), with permission from Elsevier. ${ }^{31}$

Abbreviations: $\mathrm{Cl}$, confidence interval; $\mathrm{HR}$, hazard ratio.

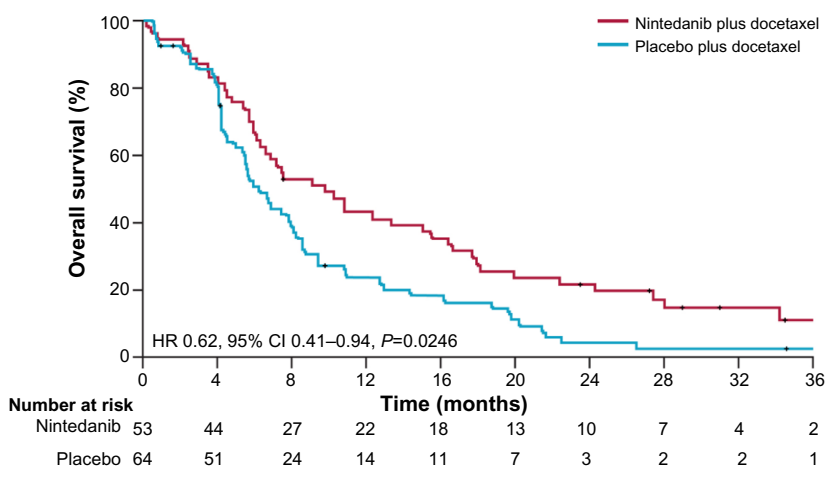

Figure 3 Kaplan-Meier curves for patients treated with nintedanib plus docetaxel or placebo plus docetaxel. Exploratory analysis in patients with adenocarcinoma histology and progressive disease as best response to first-line therapy.

Abbreviations: $\mathrm{Cl}$, confidence interval; $\mathrm{HR}$, hazard ratio.

advanced stage IIIB/IV or recurrent, non-squamous NSCLC; the primary endpoint was progression-free survival based on independent central review. ${ }^{32}$

A preplanned futility analysis was performed after $50 \%$ of progression-free survival events for the primary analysis had been observed (approximately 356 events). ${ }^{32}$ The futility analysis suggested that the study was indeed futile and that the primary endpoint was unlikely to be met; no safety concerns were raised. ${ }^{32}$ Consequently, enrollment was halted prematurely after 713 of 1,300 planned patients had entered the study. Ongoing patients were unblinded and follow-up was continued per protocol. ${ }^{32}$

Analysis of the primary endpoint, conducted after 498 progression-free survival events (database lock, July 9, 2012), showed that despite the study being stopped prematurely, the primary endpoint of centrally reviewed progression-free survival was met. ${ }^{34}$ The median progression-free survival was significantly improved in the nintedanib plus pemetrexed group compared with the placebo plus pemetrexed group (4.4 months versus 3.6 months; HR 0.83 , 95\% CI 0.70-0.99; $P=0.0435) .{ }^{34}$ The median overall survival was not significantly different between the groups (12.2 months versus 12.7 months; HR 1.03, 95\% CI 0.85-1.24; $P=0.7921) .{ }^{32}$

\section{Safety and tolerability}

Nintedanib plus docetaxel was associated with a manageable safety profile in the LUME-Lung 1 trial. ${ }^{31}$ Grade 3 or worse adverse events that were more common in the nintedanib plus docetaxel group than in the placebo plus docetaxel group were diarrhea (6.6\% versus $2.6 \%)$ and reversible increases in the liver enzymes alanine aminotransferase $(7.8 \%$ versus $0.9 \%)$ and aspartate aminotransferase $(3.4 \%$ versus $0.5 \%){ }^{31}$ Most adverse events were manageable with supportive treatment or dose reduction. ${ }^{31}$ 
At least one dose reduction of nintedanib or placebo was required by $18.6 \%$ and $6.3 \%$ of patients in the nintedanib plus docetaxel group and the placebo plus docetaxel group, respectively. ${ }^{31}$ Gastrointestinal adverse events and increases in liver enzymes accounted for most of the nintedanib dose reductions. ${ }^{31}$ Docetaxel dose reductions were needed for $15.6 \%$ and $11.9 \%$ of patients in the nintedanib plus docetaxel group and the placebo plus docetaxel group, respectively. ${ }^{31}$ Hematologic adverse events were the main reason for docetaxel dose reduction. ${ }^{31}$ The intensity of docetaxel dosing was similar in both treatment groups, indicating that nintedanib did not affect planned docetaxel dosing. ${ }^{31}$ The incidence of adverse events commonly associated with antiangiogenic agents, such as hypertension, bleeding, and gastrointestinal perforation, was similar in both groups. ${ }^{31}$

Nintedanib also had an acceptable and manageable safety profile in combination with pemetrexed, with the most frequent adverse events being reversible increases in aspartate aminotransferase and alanine aminotransferase and gastrointestinal events. ${ }^{32}$

\section{Patient perspectives}

Patient quality of life was assessed in the LUME-Lung 1 study. ${ }^{35}$ Prespecified symptoms of interest (cough, dyspnea, and pain) were assessed throughout the study using patientreported outcome measures, ie, the 30-item European Organisation for Research and Treatment of Cancer Core Quality of Life Questionnaire and its 13-item, lung cancer-specific supplement. ${ }^{35}$

Patients' global health status/quality of life was generally maintained with nintedanib plus docetaxel relative to placebo plus docetaxel, indicating that addition of nintedanib to docetaxel had no detrimental effect on quality of life. ${ }^{35}$ No differences in time to deterioration for cough, dyspnea, or pain were observed between the two treatment arms in the overall patient population. ${ }^{35}$ In patients with adenocarcinoma histology tumors, times to deterioration of quality of life rating (HR $0.82,95 \%$ CI $0.68-1.00 ; P=0.0470)$ and development of pain in the arm or shoulder (HR 0.79, 95\% CI $0.63-1.00 ; P=0.0470$ ) were significantly longer with nintedanib plus docetaxel than with placebo plus docetaxel, as was time to deterioration of gastrointestinal symptoms: diarrhea (HR 1.86, 95\% CI 1.51-2.29; $P<0.0001$ ), nausea (HR 1.27, 95\% CI 1.03-1.57; $P=0.0262$ ), and vomiting (HR $1.42,95 \%$ CI $1.11-1.81 ; P=0.0047) .{ }^{35}$ These findings indicate that the significant overall survival benefit observed with the addition of nintedanib to docetaxel therapy in patients with adenocarcinoma was achieved with no detrimental effect on patient self-reported quality of life. ${ }^{35}$

\section{Translational impact}

To date, no clinically applicable predictive biomarkers have been identified for antiangiogenic treatments in oncology. ${ }^{36}$ Predictive biomarkers that have been investigated include physiological parameters such as hypertension, circulating markers such as VEGF, soluble VEGF receptor or placental growth factor, genetic markers, and functional tumor imaging. ${ }^{36}$ Even the prospective ABIGAIL study, which comprehensively investigated the correlation between plasma biomarkers and tumor response in advanced NSCLC, failed to show any correlation between angiogenic plasma biomarkers (plasma basic FGF, E-selectin, intercellular adhesion molecule-1, placental growth factor, VEGF receptor 1, and VEGF receptor 2) and tumor response to chemotherapy plus the anti-VEGF monoclonal antibody bevacizumab. ${ }^{37}$ Only low (versus high) baseline levels ofVEGF-A (the primary ligand targeted by bevacizumab) were significantly correlated with longer progression-free survival and overall survival, demonstrating the potential of VEGF-A as a prognostic/predictive biomarker of NSCLC, with further investigation warranted. ${ }^{37}$

Although potential molecular biomarkers were not evaluated in the LUME-Lung 1 and 2 studies, exploratory, hypothesis-generating analysis of data from the LUME-Lung 2 study identified time since start of first-line therapy as a potential clinical marker of treatment benefit from nintedanib plus docetaxel, and time since start of first-line therapy less than 9 months was validated as a predictive clinical biomarker of nintedanib benefit using data from both the LUME-Lung 1 and 2 trials. ${ }^{38}$

A hypothesis-generating study using gene expression and clinical outcome data derived from a large NSCLC database was conducted to explain these observations at a gene level. ${ }^{39}$ Gene set enrichment analysis led to the following hypothesis: expression of genes relevant for cell proliferation is high in lung adenocarcinomas from patients with rapid disease progression, indicating a large fraction of proliferating cells requiring high levels of oxygen and nutrients; consequently, these tumors require high levels of vascularization and contain a large fraction of immature, growth factor-dependent blood vessels; by destroying these vessels and inhibiting vascular sprouting, nintedanib in combination with docetaxel provides greater benefit to patients with adenocarcinoma and rapidly progressive disease. ${ }^{39}$

Ongoing research is continuing to look for molecular biomarkers for the nintedanib treatment effect. Additional studies are planned, which include translational approaches to 
identify more detailed mechanisms of action for nintedanib, as well as the molecular basis of response and resistance to nintedanib; data are expected within the next 2-3 years (eg, NCT02231164). ${ }^{40}$ Effective biomarkers of response will help to reduce the number of patients who experience toxicity with no improvement in their clinical outcome, an important goal in second-line therapy. Indeed, identifying molecular biomarkers that can predict the efficacy of therapy in advanced NSCLC remains a focus of substantial research efforts.

\section{Clinical impact}

Patients with advanced NSCLC who progress rapidly during first-line platinum doublet chemotherapy have a very poor prognosis and are a population with a particularly urgent, unmet need for additional effective therapeutic options. TITAN was an international, randomized, multicenter, open-label Phase III study that compared erlotinib with chemotherapy in patients with NSCLC who had progressed rapidly after up to four cycles of first-line platinum doublet chemotherapy. ${ }^{41}$ Patients were randomly assigned (1:1) by a minimization method to receive erlotinib or chemotherapy (standard docetaxel or pemetrexed at the investigators' discretion) until unacceptable toxicity, disease progression, or death. ${ }^{41}$ In this study, erlotinib did not improve overall survival in the overall population (the primary endpoint) compared with chemotherapy (median overall survival 5.3 versus 5.5 months, respectively) and in most subgroups analyzed, suggesting comparable efficacy between these agents in this difficult-to-treat patient group. ${ }^{41}$ Consequently, the improvements in overall survival observed in the LUME-Lung 1 trial for nintedanib plus docetaxeltreated patients with adenocarcinoma and poor prognosis who were either refractory to first-line therapy or had a response of very short duration are particularly clinically relevant. ${ }^{31}$

There are currently no clinical trials that have directly compared the efficacy of second-line nintedanib plus docetaxel with other approved regimens other than docetaxel alone. However, a recent network meta-analysis of nine trials, including LUME-Lung 1, has provided an indirect estimate of relative efficacy in patients with advanced or metastatic NSCLC of adenocarcinoma histology. ${ }^{42}$ Nintedanib plus docetaxel was associated with statistically significant benefit in terms of overall survival and progression-free survival over docetaxel or erlotinib monotherapy and a non-significant benefit over pemetrexed monotherapy. The estimated probability of nintedanib plus docetaxel being the best treatment in terms of overall survival was $70.4 \%$ compared with $16.4 \%$ for pemetrexed, $9.8 \%$ for docetaxel, and $3.3 \%$ for erlotinib. ${ }^{42}$
Since publication of the LUME-Lung 1 trial, a second trial has reported improvements in overall survival with the addition of an antiangiogenic agent to docetaxel, thus confirming the principle that VEGF receptor inhibition in combination with docetaxel is a valid treatment option in second-line NSCLC patients. In the multicenter, doubleblind, randomized (1:1) Phase III REVEL ( $\mathrm{n}=1,253)$ study, the addition of ramucirumab, a human immunoglobulin G1 monoclonal antibody that targets the extracellular domain of VEGF receptor 2, to docetaxel demonstrated significant improvement in the primary endpoint of median overall survival (10.5 versus 9.1 months) and median progression-free survival (4.5 versus 3.0 months) versus placebo plus docetaxel in the total population (squamous and non-squamous NSCLC patients). ${ }^{43}$ The most common grade 3 or worse adverse events were neutropenia (49\% in the ramucirumab group versus $40 \%$ in the control group), febrile neutropenia (16\% versus $10 \%)$, fatigue (14\% versus $10 \%)$, leucopenia ( $14 \%$ versus $12 \%$ ), and hypertension ( $6 \%$ versus $2 \%) .{ }^{43}$

In addition to trials in NSCLC, nintedanib has been evaluated in a number of other solid tumors, including colorectal cancer, ${ }^{44,45}$ renal cell carcinoma, ${ }^{46}$ and hepatocellular carcinoma. ${ }^{47,48}$ Following promising Phase I trials in patients with refractory colorectal cancer, the randomized (1:1) Phase III LUME-Colon 1 study is recruiting patients to assess nintedanib plus best supportive care as last-line treatment for colorectal cancer (NCT02149108). ${ }^{44,45}$ The Phase II 1199.26 study (NCT01024920) evaluated the efficacy and safety of nintedanib versus sunitinib as first-line therapy in previously untreated patients with renal cell carcinoma. ${ }^{49}$ Two Phase II studies (1199.37: NCT01004003 and 1199.39: NCT00987935) have investigated the efficacy and safety of nintedanib versus sorafenib in patients with advanced hepatocellular carcinoma. ${ }^{50,51}$ They have shown that nintedanib has efficacy that is comparable with sorafenib in patients with advanced hepatocellular carcinoma, with a manageable adverse event profile, indicating that further studies of nintedanib in hepatocellular carcinoma are warranted. ${ }^{47,48}$

A randomized (1:1) Phase II study to evaluate the efficacy and safety of nintedanib versus placebo in combination with pemetrexed/cisplatin combination chemotherapy is recruiting patients with malignant pleural mesothelioma (NCT01907100). ${ }^{49}$

In addition to the November 2014 European approval of nintedanib for the treatment of patients with NSCLC who progressed following first-line chemotherapy, ${ }^{17}$ nintedanib has been approved by the US Food and Drug Administration and has received a positive opinion from the European Medicines Agency's Committee 
for Medicinal Products for Human Use for the treatment of patients with idiopathic pulmonary fibrosis. ${ }^{52,53}$

\section{Conclusion}

The use of nintedanib in combination with docetaxel as a treatment option for patients with locally advanced, metastatic, or locally recurrent NSCLC of adenocarcinoma tumor histology after first-line chemotherapy is now an available treatment option and offers much-needed improvements in overall survival for this patient group. The safety profile of nintedanib in combination with docetaxel is manageable, giving this new treatment option great potential as an emerging combination for the management of NSCLC.

\section{Disclosure}

Medical writing assistance, supported financially by Boehringer Ingelheim Pharma GmbH \& Co KG, Ingelheim, Germany, was provided by Saira Silie (inVentiv Medical Communications, Maidenhead, UK) during preparation of this report. The authors report no other conflicts of interest in this paper.

\section{References}

1. World Health Organization: International Agency for Research on Cancer. GLOBOCAN 2012: Estimated cancer incidence, mortality and prevalence worldwide in 2012. Available from: http://globocan.iarc.fr/ Pages/fact_sheets_cancer.aspx\#. Accessed February 19, 2015.

2. National Comprehensive Cancer Network: NCCN Clinical Practice Guidelines in Oncology (NCCN Guidelines): non-small cell lung cancer. Version 4. Available from: http:/www.nccn.org/professionals/ physician_gls/pdf/nscl.pdf. Accessed February 19, 2015.

3. Aggarwal C, Somaiah N, Simon G. Antiangiogenic agents in the management of non-small cell lung cancer: where do we stand now and where are we headed? Cancer Biol Ther. 2012;13:247-263.

4. Besse B, Adjei A, Baas P, et al. 2nd ESMO Consensus Conference on Lung Cancer: non-small-cell lung cancer first-line/second and further lines of treatment in advanced disease. Ann Oncol. 2014;25: 1475-1484.

5. Reck M, Popat S, Reinmuth N, et al. Metastatic non-small-cell lung cancer (NSCLC): ESMO Clinical Practice Guidelines for diagnosis, treatment and follow-up. Ann Oncol. 2014;25:iii27-iii39.

6. Stinchcombe TE, Socinski MA. Considerations for second-line therapy of non-small cell lung cancer. Oncologist. 2008;13:28-36.

7. Gridelli C, Ardizzoni A, Ciardiello F, et al. Second-line treatment of advanced non-small cell lung cancer. J Thorac Oncol. 2008;3: 430-440.

8. Johnson DH, Fehrenbacher L, Novotny WF, et al. Randomized phase II trial comparing bevacizumab plus carboplatin and paclitaxel with carboplatin and paclitaxel alone in previously untreated locally advanced or metastatic non-small-cell lung cancer. J Clin Oncol. 2004;22: 2184-2191.

9. Fossella FV, DeVore R, Kerr RN, et al. Randomized phase III trial of docetaxel versus vinorelbine or ifosfamide in patients with advanced non-small-cell lung cancer previously treated with platinum-containing chemotherapy regimens. The TAX 320 Non-Small Cell Lung Cancer Study Group. J Clin Oncol. 2000;18:2354-2362.

10. Hanna N, Shepherd FA, Fossella FV, et al. Randomized phase III trial of pemetrexed versus docetaxel in patients with non-small-cell lung cancer previously treated with chemotherapy. J Clin Oncol. 2004;22: 1589-1597.
11. Thatcher N, Chang A, Parikh P, et al. Gefitinib plus best supportive care in previously treated patients with refractory advanced non-small-cell lung cancer: results from a randomised, placebo-controlled, multicentre study (Iressa Survival Evaluation in Lung Cancer). Lancet. 2005;366: $1527-1537$

12. Herbst RS, Sun Y, Eberhardt WE, et al. Vandetanib plus docetaxel versus docetaxel as second-line treatment for patients with advanced non-small-cell lung cancer (ZODIAC): a double-blind, randomised, phase 3 trial. Lancet Oncol. 2010;11:619-626.

13. de Boer $\mathrm{RH}$, Arrieta $\mathrm{O}$, Yang $\mathrm{CH}$, et al. Vandetanib plus pemetrexed for the second-line treatment of advanced non-small-cell lung cancer: a randomized, double-blind phase III trial. J Clin Oncol. 2011;29: 1067-1074.

14. Natale RB, Thongprasert S, Greco FA, et al. Phase III trial of vandetanib compared with erlotinib in patients with previously treated advanced non-small-cell lung cancer. J Clin Oncol. 2011;29:1059-1066.

15. Herbst RS, Ansari R, Bustin F, et al. Efficacy of bevacizumab plus erlotinib versus erlotinib alone in advanced non-small-cell lung cancer after failure of standard first-line chemotherapy (BeTa): a double-blind, placebo-controlled, phase 3 trial. Lancet. 2011;377: 1846-1854.

16. Garassino MC, Martelli O, Broggini M, et al. Erlotinib versus docetaxel as second-line treatment of patients with advanced non-small-cell lung cancer and wild-type EGFR tumours (TAILOR): a randomised controlled trial. Lancet Oncol. 2013;14:981-988.

17. European Medicines Agency. Vargatef: summary of product characteristics. 2014. Available from: http://ec.europa.eu/health/ documents/community-register/2014/20141121130020/anx_130020_ en.pdf. Accessed March 19, 2015.

18. Hilberg F, Roth GJ, Krssak M, et al. BIBF 1120: triple angiokinase inhibitor with sustained receptor blockade and good antitumor efficacy. Cancer Res. 2008;68:4774-4782.

19. Roth GJ, Heckel A, Colbatzky F, et al. Design, synthesis, and evaluation of indolinones as triple angiokinase inhibitors and the discovery of a highly specific 6-methoxycarbonyl-substituted indolinone (BIBF 1120). J Med Chem. 2009;52:4466-4480.

20. Roth GJ, Binder R, Colbatzky F, et al. Nintedanib: from discovery to the clinic. J Med Chem. 2015;58:1053-1063.

21. Hilberg F, Brandstetter I. Efficacy of BIBF 1120, a potent triple angiokinase inhibitor, in models of human non-small cell lung cancer is augmented by chemotherapy. Eur J Cancer. 2007;2:S380.

22. Kutluk Cenik B, Ostapoff KT, Gerber DE, Brekken RA. BIBF 1120 (nintedanib), a triple angiokinase inhibitor, induces hypoxia but not EMT and blocks progression of preclinical models of lung and pancreatic cancer. Mol Cancer Ther. 2013;12:992-1001.

23. Stopfer P, Rathgen K, Bischoff D, et al. Pharmacokinetics and metabolism of BIBF 1120 after oral dosing to healthy male volunteers. Xenobiotica. 2011;41:297-311.

24. Mross K, Stefanic M, Gmehling D, et al. Phase I study of the angiogenesis inhibitor BIBF 1120 in patients with advanced solid tumors Clin Cancer Res. 2010;16:311-319.

25. Lee CP, Taylor NJ, Attard G, et al. A phase I study of BIBF 1120, an orally active triple angiokinase inhibitor (VEGFR, PDGFR, FGFR) given continuously to patients with advanced solid tumours, incorporating dynamic contrast enhanced magnetic resonance imaging (DCE-MRI). J Clin Oncol. 2006;24:3015.

26. Okamoto I, Kaneda H, Satoh T, et al. Phase I safety, pharmacokinetic, and biomarker study of BIBF 1120, an oral triple tyrosine kinase inhibitor in patients with advanced solid tumors. Mol Cancer Ther. 2010;9: 2825-2833.

27. Doebele RC, Conkling P, Traynor AM, et al. A phase I, open-label dose-escalation study of continuous treatment with BIBF 1120 in combination with paclitaxel and carboplatin as first-line treatment in patients with advanced non-small-cell lung cancer. Ann Oncol. 2012;23: 2094-2102.

28. Ellis PM, Kaiser R, Zhao Y, et al. Phase I open-label study of continuous treatment with BIBF 1120, a triple angiokinase inhibitor, and pemetrexed in pretreated non-small cell lung cancer patients. Clin Cancer Res. 2010;16:2881-2889. 
29. Okamoto I, Miyazaki M, Takeda M, et al. Tolerability of nintedanib (BIBF 1120) in combination with docetaxel: a Phase 1 study in Japanese patients with previously treated non-small-cell lung cancer. $J$ Thorac Oncol. 2015;10:346-352.

30. Reck M, Kaiser R, Eschbach C, et al. A phase II double-blind study to investigate efficacy and safety of two doses of the triple angiokinase inhibitor BIBF 1120 in patients with relapsed advanced non-small-cell lung cancer. Ann Oncol. 2011;22:1374-1381.

31. Reck M, Kaiser R, Mellemgaard A, et al. Docetaxel plus nintedanib versus docetaxel plus placebo in patients with previously treated non-small-cell lung cancer (LUME-Lung 1): a phase 3, double-blind, randomised controlled trial. Lancet Oncol. 2014;15:143-155.

32. Hanna N, Kaiser R, Kim J-H, et al. LUME-Lung 2: a multicenter, randomized, double-blind, phase III study of nintedanib plus pemetrexed versus placebo plus pemetrexed in patients with advanced non-squamous non-small cell lung cancer after failure of first line chemotherapy. Presented at the Annual Meeting of the American Society of Clinical Oncology, Chicago, IL, USA, May 31 to June 4, 2013.

33. Mellemgaard A, Kaiser R, Douillard JY, et al. Analysis of overall survival in adenocarcinoma NSCLC patients receiving 2nd line combination treatment with nintedanib (BIBF 1120) + docetaxel in the LUMELung 1 trial: A randomized, double-blind, placebo-controlled phase 3 study. Presented at the The European Cancer Congress, Amsterdam, The Netherlands, September 27 to October 1, 2013.

34. Hocke J, Glomb P, Kaiser R, Barrueco J, Gaschler-Markefski B. Retrospective evaluation of the futility analysis in LUME-Lung 2, a phase III trial of nintedanib (BIBF 1120) plus pemetrexed for second line NSCLC patients. Presented at the International Society for Clinical Biostatistics 35th Annual Meeting, Vienna, Austria, August 24-28, 2014.

35. Novello S, Kaiser R, Mellemgaard A, et al. Analysis of patient-reported outcomes from the LUME-Lung 1 trial: a randomized, double-blind, placebo-controlled, Phase III study of second-line nintedanib in patients with advanced non-small cell lung cancer. Eur J Cancer. 2015;51: $317-326$.

36. Jubb AM, Harris AL. Biomarkers to predict the clinical efficacy of bevacizumab in cancer. Lancet Oncol. 2010;11:1172-1183.

37. Mok T, Gorbunova V, Juhasz E, et al. A correlative biomarker analysis of the combination of bevacizumab and carboplatin-based chemotherapy for advanced nonsquamous non-small-cell lung cancer: results of the phase II randomized ABIGAIL study (BO21015). J Thorac Oncol. 2014;9:848-855.

38. Kaiser R, Barrueco J, Reck M, et al. Identification of a clinical biomarker for second-line combination treatment with nintedanib in adenocarcinoma non-small cell lung cancer (NSCLC) patients in two phase III trials. Presented at the European Cancer Congress (ECCO-ESMO-ESTRO), Amsterdam, The Netherlands, September 27 to October 1, 2013.

39. Hilberg F, Haslinger C, Garin-Chesa P, Adolf GR. Molecular correlates of clinical benefit from antiangiogenic therapy for patients with lung adenocarcinoma: a hypothesis. J Clin Oncol. 2014;32 Suppl: Abstr e22080.

40. Boehringer Ingelheim. LUME-Columbus: nintedanib plus docetaxel in advanced non-small cell lung cancer with translational research. 2015. Available from: https:/clinicaltrials.gov/ct2/show/NCT02231164. Accessed March 19, 2015.

Biologics: Targets \& Therapy

\section{Publish your work in this journal}

Biologics: Targets \& Therapy is an international, peer-reviewed journal focusing on the patho-physiological rationale for and clinical application of Biologic agents in the management of autoimmune diseases, cancers or other pathologies where a molecular target can be identified. This journal is indexed on PubMed Central, CAS, EMBase, Scopus
41. Ciuleanu T, Stelmakh L, Cicenas S, et al. Efficacy and safety of erlotinib versus chemotherapy in second-line treatment of patients with advanced, non-small-cell lung cancer with poor prognosis (TITAN): a randomised multicentre, open-label, phase 3 study. Lancet Oncol. 2012;13:300-308.

42. Popat S, Mellemgaard A, Fahrbach K, et al. Nintedanib plus docetaxel as second-line therapy in patients with non-small-cell lung cancer: a network meta-analysis. Future Oncol. 2015;11:409-420.

43. Garon EB, Ciuleanu TE, Arrieta O, et al. Ramucirumab plus docetaxel versus placebo plus docetaxel for second-line treatment of stage IV non-small-cell lung cancer after disease progression on platinum-based therapy (REVEL): a multicentre, double-blind, randomised phase 3 trial. Lancet. 2014;384:665-673.

44. Van Cutsem E, Prenen H, Guillen-Ponce C, et al. A phase I/II, openlabel, randomised study of BIBF 1120 plus mFOLFOX6 compared to bevacizumab plus mFOLFOX6 in patients with metastatic colorectal cancer. Eur J Cancer. 2011;47:8-9.

45. Van Cutsem E, Tabernero J, Yoshino T, et al. LUME-Colon 1: doubleblind, randomised phase III study of nintedanib plus best supportive care (BSC) versus placebo plus BSC in patients with refractory colorectal cancer. Ann Oncol. 2014;25 Suppl 4:iv208 (Abstr 610TiP).

46. Eisen T, Shparyk Y, Jones R, et al. Phase II efficacy and safety study of nintedanib versus sunitinib in previously untreated renal cell carcinoma (RCC) patients. J Clin Oncol. 2013;31:Abstr 4506.

47. Yen C, Kim TY, Feng YH, et al. Efficacy and safety of nintedanib vs sorafenib in Asian patients with advanced hepatocellular carcinoma (HCC): a randomised Phase II trial. Eur J Cancer. 2013;49:S612 (Abstr 2580)

48. Palmer D, Peck-Radosavljevic M, Ma YT, et al. Open-label, phase I/ randomized phase II trial of the triple angiokinase inhibitor, nintedanib, versus sorafenib in previously untreated patients with advanced hepatocellular carcinoma (HCC). J Clin Oncol. 2013;31:Abstr TPS4160.

49. Boehringer Ingelheim. Compare safety and efficacy of nintedanib (BIBF 1120) versus sunitinib. 2014. http://clinicaltrials.gov/ct2/show/ NCT01024920. Accessed April 01, 2015.

50. Palmer D, Ma YT, Peck-Radosavljevic M, et al. Randomized phase II trial comparing the efficacy and safety of nintedanib versus sorafenib in patients with advanced hepatocellular carcinoma (HCC). Presented at the ASCO Gastrointestinal Cancers Symposium, San Francisco, CA, USA, January 15-17, 2015.

51. Cheng AL, Yen C, Kim JH, et al. Efficacy and safety of nintedanib versus sorafenib in Asian patients with advanced hepatocellular carcinoma (HCC): a randomized phase II trial. Presented at the ASCO Gastrointestinal Cancers Symposium, San Francisco, CA, USA, January 15-17, 2015.

52. US Food and Drug Administration. FDA approves Ofev to treat idiopathic pulmonary fibrosis. Available from: http://www.fda.gov/ newsevents/newsroom/pressannouncements/ucm 418994.htm. Accessed February 19, 2015.

53. European Medicines Agency. Summary of opinion (initial authorisation): Ofev (nintedanib). 2014. Available from: http://www.ema. europa.eu/docs/en_GB/document_library/Summary_of_opinion_-Initial_authorisation/human/003821/WC500177632.pdf. Accessed March 19, 2015.

and the Elsevier Bibliographic databases. The manuscript management system is completely online and includes a very quick and fair peerreview system, which is all easy to use. Visit http://www.dovepress com/testimonials.php to read real quotes from published authors.

\section{Dovepress}

